\title{
Detection of Aberrant Right Subclavian Artery by PET/CT
}

\author{
Zaid Al-faham, Andrew C. Boyer, Ching-Yee Oliver Wong, and Dafang Wu \\ Department of Diagnostic Radiology and Molecular Imaging, Oakland University William Beaumont School of Medicine \\ and Health System, Royal Oak, Michigan
}

\begin{abstract}
An aberrant right subclavian artery can be diagnosed by PET/CT, as demonstrated in this case of a $70-y$-old man undergoing $\mathrm{PET} / \mathrm{CT}$ for staging of squamous cell carcinoma of the right lung. It is important to report this finding during the evaluation of oncologic patients, to prevent severe complications that may arise from various oncologic interventions.
\end{abstract}

Key Words: correlative imaging; PET; PET/CT; aberrant right subclavian artery

J Nucl Med Technol 2015; 43:129-130

DOI: 10.2967/jnmt.114.137703

$\mathbf{T}$

o our knowledge, this is the first case in the literature that describes the identification of an aberrant right subclavian artery on PET/CT. It is important to identify the presence of an aberrant right subclavian artery in order to prevent an erroneous diagnosis, an unnecessary imaging workup, and rare severe complications.

\section{CASE REPORT}

A PET/CT scan was ordered for staging in a 70-y-old man with a history of squamous cell carcinoma of the right lung. The imaging was performed on a dedicated 16-slice body PET/CT scanner (Discovery DST; GE Healthcare). Ninety minutes after intravenous administration of $619.01 \mathrm{MBq}$ $(16.73 \mathrm{mCi})$ of ${ }^{18} \mathrm{~F}-\mathrm{FDG}$ under fasting conditions, sequential unenhanced CT images and then PET images were acquired. For the PET component, imaging was performed at each bed position for $3 \mathrm{~min}$, using 6-8 bed positions to cover the entire body. A 3-dimensional high-sensitivity mode was used, with an axial field of view of $15 \mathrm{~cm}$ in a $256 \times 256$ matrix. The images were reconstructed iteratively on a $128 \times 128$ matrix using an ordered-subsets expectation maximization algorithm for 30 subsets and 2 iterations, with a 7.0-mm postreconstruc-

\footnotetext{
Received Jan. 19, 2014; revision accepted Jun. 26, 2014.

For correspondence or reprints contact: Zaid Al-faham, William Beaumont Hospital, 3601 W. 13 Mile Rd., Royal Oak, Ml 48073.

E-mail: zaidfaham@gmail.com

Published online Aug. 14, 2014.

COPYRIGHT (c) 2015 by the Society of Nuclear Medicine and Molecular Imaging, Inc.
}

tion filter. For the CT component, the following imaging parameters were used: $140 \mathrm{kVp}, 120-200 \mathrm{~mA}, 0.8 \mathrm{~s}$ per CT rotation, a pitch of $1.75: 1$, a detector configuration of $16 \times 1.25 \mathrm{~mm}$, a $3-\mathrm{mm}$ slice thickness, and oral contrast material only. The PET scan was positive for an ${ }^{18} \mathrm{~F}-\mathrm{FDG}-$ avid soft-tissue lesion in the right middle lobe and for a low-density lesion in the spleen. An aberrant right subclavian artery was also visualized posterior to the esophagus (Fig. 1).

\section{DISCUSSION}

An aberrant right subclavian artery is a rare congenital anomaly with a reported incidence of less than 1\%-2\% (1$4)$. It results from regression of the right fourth aortic arch and persistence of the dorsal aorta $(1,3,4)$. Patients with an aberrant right subclavian artery are mostly asymptomatic. However, dysphagia, stridor, thoracic pain, and cough are the most common symptoms (1). In more than $80 \%$ of cases, the aberrant right subclavian artery is posterior to the esophagus, making the artery susceptible to extrinsic compression-such as by placement of nasogastric or endotracheal tubes - that could lead to a right subclavian artery esophageal fistula causing severe upper gastrointestinal bleeding (2). It is essential to identify an aberrant right subclavian artery so that it may be included in the differential diagnosis of dysphagia in children as it may be overlooked by endoscopy (3). Given that there are no important vessels in the area between the esophagus and the spine at the level above the aortic arch, it is considered a safe area in patients undergoing esophageal dissection and is often uninvestigated before surgery. Thus, the surgeon should consider this condition because a transected aberrant right subclavian artery could lead to upper-limb ischemia (4). Barium contrast imaging of the esophagus, MR imaging, $\mathrm{CT}$, and angiography are used to diagnose an aberrant right subclavian artery and its complications (1). In this case, PET/CT detected this condition as well. Surgery is reserved for symptomatic patients or those with an aberrant right subclavian artery aneurysm (1).

\section{CONCLUSION \\ ${ }^{18}$ F-FDG PET/CT can reveal an aberrant right subcla- vian artery. It is important to report this finding during}


FIGURE 1. Maximum-intensity-projection PET images (A) and fusion PET/CT images (B; sagittal view [left], coronal view [middle], and axial view [right]). $\mathrm{E}=$ esophagus; $\mathrm{O}=$ origin of aberrant subclavian artery from aorta; $\mathrm{S}=$ subclavian artery.

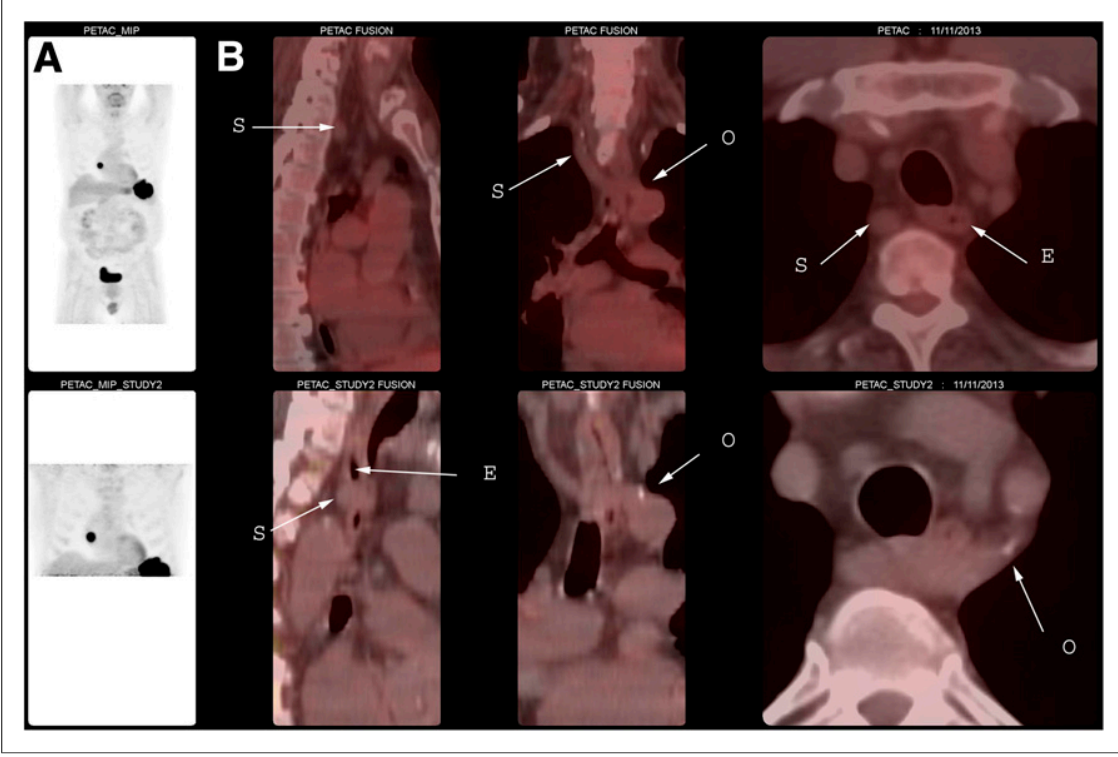

the evaluation of oncologic patients, to prevent severe complications that may arise from various oncologic interventions.

\section{DISCLOSURE}

No potential conflict of interest relevant to this article was reported.

\section{REFERENCES}

1. Atay Y, Engin C, Posacioglu H, et al. Surgical approaches to the aberrant right subclavian artery. Tex Heart Inst J. 2006;33:477-481.

2. Millar A, Rostom A, Rasuli P, Saloojee N. Upper gastrointestinal bleeding secondary to an aberrant right subclavian artery-esophageal fistula: a case report and review of the literature. Can J Gastroenterol. 2007;21:389-392.

3. Ulger Z, Ozyürek AR, Levent E, Gürses D, Parlar A. Arteria lusoria as a cause of dysphagia. Acta Cardiol. 2004;59:445-447.

4. Lin Y, Li M, Liu X. Aberrant right subclavian artery in video-assisted thoracoscopic esophagectomy. J Thorac Dis. 2013;5:E137-E139. 